\title{
Connection and speed control in railway systems - A model predictive control $\operatorname{approach}^{*}$
}

\author{
B. De Schutter and T.J.J. van den Boom
}

If you want to cite this report, please use the following reference instead:

B. De Schutter and T.J.J. van den Boom, "Connection and speed control in railway systems - A model predictive control approach," Proceedings of the 6th International Workshop on Discrete Event Systems (WODES'02) (M. Silva, A. Giua, and J.M. Colom, eds.), Zaragoza, Spain, pp. 49-54, Oct. 2002. 


\title{
Connection and Speed Control in Railway Systems - A Model Predictive Control Approach
}

\author{
B. De Schutter and T.J.J. van den Boom \\ Control Systems Engineering, Fac. ITS, Delft University of Technology \\ P.O. Box 5031, 2600 GA Delft, The Netherlands \\ E-mail: \{b.deschutter,t.j.j.vandenboom\}@its.tudelft.nl
}

\begin{abstract}
We further enhance our previous results on model predictive control (MPC) for railway systems with both hard and soft connection constraints, i.e., railway systems where, if necessary, some connections may be broken (but then a penalty is incurred). In this paper we extend the previous model by also including variable traveling times, which offers an extra degree of freedom for control. We present an MPC framework for railway systems, where the main aim of the control is to recover from delays in an optimal way by breaking connections and/or letting some trains run faster than usual (both at a cost). In general, the MPC control design problem for railway systems leads to a nonlinear nonconvex optimization problem, but we show that the optimal MPC strategy can be computed using extended linear complementarity problems or integer programming.
\end{abstract}

\section{Introduction and overview}

Currently, model predictive control (MPC) [10, 12] is one of the most popular advanced control design techniques in the process industry. MPC is a model-based control design method that allows the inclusion of constraints on inputs and outputs, and that can handle changes in the system parameters by using a moving horizon approach, in which the model and the control strategy are updated continuously. In this paper we extend and adapt the MPC framework to railway (or subway) networks. Note that the approach can also be used for, e.g., logistic systems. The proposed MPC approach has the following ingredients (which are also present in conventional MPC): a prediction horizon, a receding horizon procedure, and a regular update of the model and re-computation of the optimal control input.

In [5] we have already extended MPC to a class of discrete event systems with "hard" synchronization constraints, i.e., synchronization constraints that should always be met. However, railway networks are characterized by the occurrence of both soft and hard synchronization constraints. A typical example of a hard synchronization constraint in a railway context is when a train should give a guaranteed connection to another train. However, in some cases (e.g., if there are delays) we could allow a train to depart although not all trains to which it should give connection according to the schedule have arrived at the station: if some of these trains have a too large delay, then it is sometimes better - from a global performance viewpoint — to let the train depart anyway in order to prevent an accumulation of delays in the network. Of course, missed connections lead to a penalty due to dissatisfied passengers or due to compensations that have to be paid. Synchronization constraints that may be broken (but at a cost) are called soft synchronization constraints. In $[4,6]$ we have presented a modeling framework for railway systems and other discrete event systems with soft and hard synchronization constraints. In this paper, which is an improved and significantly extended version of $[4,6]$, we consider an extra degree of freedom for the control by letting trains run faster than their nominal speed if necessary. Of course, this control action will also lead to extra costs (due to increased energy consumption or faster wear of the material).

Other work in connection with modeling and control of railway networks in a discrete event systems context can be found in $[1,7,9,11]$. The main contribution and difference of our approach compared to the work by other researchers is that we present an on-line model-based control design approach that includes hard and soft synchronization constraints, that is an extension of the popular MPC approach, and that uses optimization with continuous variables. Note that we do not consider re-routing or adapting the timetable.

\section{A modeling framework for railway net- works}

Consider a railway operations system, the nominal operation of which follows a timetable with a period $T$. We 


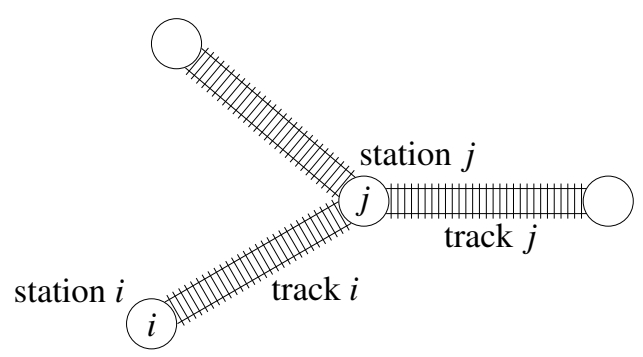

Figure 1. A part of a railway network.

assume that all the trains follow a pre-scheduled route. Let $n$ be the number of tracks in the network. Each track has a number and a virtual train allocated to it. For the sake of simplicity we will say "(virtual) train $j$ " to denote the (physical) train on track $j$, and "station $j$ " to denote the station at the beginning of track $j$ (cf. Figure 1). Let $x_{j}(k)$ be the time instant at which train $j$ departs from station $j$ during the $k$ th operation cycle. Let $d_{j}(k)$ be the departure time for this train according to the timetable, and let $a_{j}(k)$ be the actual traveling time of this train.

The set $\mathscr{C}_{j}(k)$ of trains to which the train on track $j$ gives a connection during cycle $k$ can be divided into a set of hard connections $\mathscr{C}_{j}^{\text {hard }}(k)$ (e.g., if the train on track $i$ and the train on track $j$ are physically the same train, or if it is a very important connection that should be guaranteed at all costs) and a set of soft connections $\mathscr{C}_{j}^{\text {soft }}(k)$ (e.g., local trains to which the train $j$ should give connection, but if the local train $i \in \mathscr{C}_{j}^{\text {soft }}(k)$ has a too large delay, then the connection may be broken; however, in that case a maximal cost $c_{i, j}^{\text {broken }}(k)$ is associated with the broken connection). We also define a minimum connection time $t_{i, j}^{\min }(k)$ for passengers to get from train $i$ to train $j$ for each train $i \in \mathscr{C}_{j}(k)$.

We have the following constraints for the departure time $x_{j}(k)$ of train $j$ in the cycle $k$ :

- timetable constraint: Train $j$ should not depart before the departure time given in the timetable has passed:

$$
x_{j}(k) \geqslant d_{j}(k)
$$

- hard synchronization constraints:

If there is another train $i$ to which train $j$ should give a hard connection, then train $j$ may only depart if train $i$ has arrived and the passengers have gotten enough time to get out of the train or to change trains. So

$$
x_{j}(k) \geqslant x_{i}\left(k-\delta_{i, j}(k)\right)+a_{i}(k)+t_{i, j}^{\min }(k)
$$

for each $i \in \mathscr{C}_{j}^{\text {hard }}(k)$, where $\delta_{i, j}(k)$ denotes the cycle delay between train $i$ and train $j$ for the $k$ th cycle, i.e., train $j$ in cycle $k$ gives connection to the train $i \in \mathscr{C}_{j}(k)$ that departed from its station in cycle $k-\delta_{i, j}(k)$.
- soft synchronization constraints:

If the connection takes place, we have a constraint that is similar to (2). If the connection with train $i \in \mathscr{C}_{j}^{\text {soft }}(k)$ is broken, train $j$ departs before train $i$ has arrived and all the passengers have gotten the time to change trains. So for each train $i \in \mathscr{C}_{j}^{\text {soft }}(k)$ we have

$$
x_{j}(k) \geqslant x_{i}\left(k-\delta_{i, j}(k)\right)+a_{i}(k)+t_{i, j}^{\min }(k)
$$

if the connection takes place,

$$
x_{j}(k)<x_{i}\left(k-\delta_{i, j}(k)\right)+a_{i}(k)+t_{i, j}^{\min }(k)
$$

if the connection is broken.

If we introduce a control variable $u_{i, j}(k) \geqslant 0$, then we can combine these equations into

$$
x_{j}(k) \geqslant x_{i}\left(k-\delta_{i, j}(k)\right)+a_{i}(k)+t_{i, j}^{\min }(k)-u_{i, j}(k)
$$

where $u_{i, j}(k)$ can be used to guarantee or to break a connection.

Since we let a train depart as soon as all connection conditions are satisfied, (1) - (3) leads to:

$$
\begin{aligned}
x_{j}(k)= & \max \left(d_{j}(k), \max _{i \in \mathscr{C}_{j}^{\text {hard }}(k)}\left(x_{i}\left(k-\delta_{i, j}(k)\right)+a_{i}(k)+t_{i, j}^{\min }(k)\right),\right. \\
& \left.\max _{i \in \mathscr{C}_{j}^{\text {soft }}(k)}\left(x_{i}\left(k-\delta_{i, j}(k)\right)+a_{i}(k)+t_{i, j}^{\min }(k)-u_{i, j}(k)\right)\right)
\end{aligned}
$$

Apart from breaking soft connections if necessary, we also consider adapting the speed of the train as an additional control measure. Of course, this will lead to extra costs (due to increased energy consumption or faster wear of the material). Let $a_{i}^{\min }(k)$ be the minimal traveling time (i.e., assuming that the train runs at full speed) from station $i$ to station $j$ for each train $i \in \mathscr{C}_{j}(k)$, and let $a_{i}^{\text {nom }}(k)$ be the nominal traveling time (i.e., assuming that the train runs at its most economical speed, while still guaranteeing that the timetable is met within a given safety margin). We introduce an extra control variable $v_{i}(k)$ to modify the traveling time $a_{i}(k)$ on track $i$ (i.e., the track from station $i$ to station $j$ ) in cycle $k$ :

$$
a_{i}(k)=a_{i}^{\text {nom }}(k)-v_{i}(k)
$$

with the constraint $0 \leqslant v_{i}(k) \leqslant a_{i}^{\text {nom }}(k)-a_{i}^{\min }(k)$ for all $k, i$. This results in:

$$
\begin{aligned}
& x_{j}(k)=\max \left(d_{j}(k),\right. \\
& \max _{i \in \mathscr{C}_{j}^{\text {hard }}(k)}\left(x_{i}\left(k-\delta_{i, j}(k)\right)+a_{i}^{\text {nom }}(k)-v_{i}(k)+t_{i, j}^{\min }(k)\right), \\
& \left.\max _{i \in \mathscr{C}_{j}^{\text {soft }}(k)}\left(x_{i}\left(k-\delta_{i, j}(k)\right)+a_{i}^{\text {nom }}(k)-v_{i}(k)+t_{i, j}^{\min }(k)-u_{i, j}(k)\right)\right) .
\end{aligned}
$$

\section{The railway MPC problem}

Define $t_{i, j}^{\text {slack }}(k)$ as the slack time of the arrival of train $i \in$ $\mathscr{C}_{j}^{\text {soft }}(k)$ at station $j$ (transit time $t_{i, j}^{\min }(k)$ included) w.r.t. the 


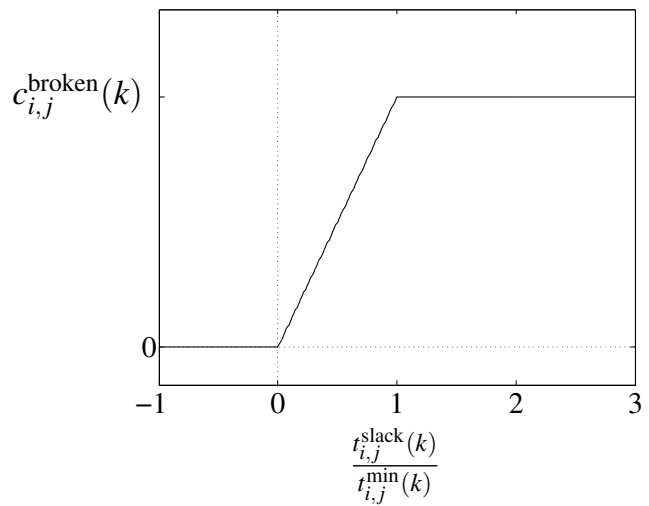

Figure 2. The piecewise-affine cost function $J^{\text {broken }}$ for broken connections defined by (6).

actual departure time of train $j$ in cycle $k$ :

$$
t_{i, j}^{\text {slack }}(k)=x_{i}\left(k-\delta_{i, j}(k)\right)+a_{i}^{\text {nom }}(k)-v_{i}(k)+t_{i, j}^{\min }(k)-x_{j}(k) .
$$

If $t_{i, j}^{\text {slack }}(k) \leqslant 0$, the connection between train $i$ and $j$ is completely guaranteed (with enough time for the passengers to change trains). If $t_{i, j}^{\text {slack }}(k)>t_{i, j}^{\min }(k)$, train $j$ leaves the station before the arrival of train $i$. If $0<t_{i, j}^{\text {slack }}(k) \leqslant t_{i, j}^{\min }(k)$, the connection is partly guaranteed (i.e., fast-running passengers get the connection, but slower ones may lose it). Therefore, we define the cost of a broken connection as the following piecewise-affine function (cf. Figure 2):

$$
J_{i, j}^{\text {broken }}(k)= \begin{cases}0 & \text { if } t_{i, j}^{\text {slack }}(k) \leqslant 0, \\ \frac{c_{i, j}^{\text {broken }}(k)}{t_{i, j}^{\min }(k)} t_{i, j}^{\text {slack }}(k) & \text { if } 0<t_{i, j}^{\text {slack }}(k) \leqslant t_{i, j}^{\min }(k), \\ c_{i, j}^{\text {roken }}(k) & \text { if } t_{i, j}^{\text {slack }}(k)>t_{i, j}^{\min }(k) .\end{cases}
$$

We use the following quadratic cost function to express the extra costs related to increasing the speed of the train on track $i$ in cycle $k$ :

$$
J_{i}^{\text {speed }}(k)=c_{i}^{\text {speed }}(k) v_{i}^{2}(k) .
$$

where $c_{i}^{\text {speed }}(k)$ characterizes the extra cost per squared unit traveling time decrease for the train on track $i$ in cycle $k$.

In each MPC cycle we consider the evolution of the system over a given prediction horizon $N_{\mathrm{p}}$. We define the following cost function over the period consisting of cycles $k$ up to $k+N_{\mathrm{p}}-1$ :

$$
\begin{aligned}
J_{\text {cost }}(k)= & \sum_{l=0}^{N_{\mathrm{p}}-1} \sum_{j=1}^{n} c_{j}^{\text {off }}(k+l)\left(\hat{x}_{j}(k+l \mid k)-d_{j}(k+l)\right)^{2}+ \\
& \lambda \sum_{l=0}^{N_{\mathrm{p}}-1} \sum_{j=1}^{n} \sum_{i \in \mathscr{C}_{j}^{\text {sot }}(k+l)} J_{i, j}^{\text {broken }}(k+l)+ \\
& \mu \sum_{l=0}^{N_{\mathrm{p}}-1} \sum_{j=1}^{n} \sum_{i \in \mathscr{C}_{j}(k+l)} J_{i}^{\text {speed }}(k+l)
\end{aligned}
$$

where $\lambda, \mu>0$ are weighting factors, $c_{j}^{\text {off }}(k)$ is the offschedule cost per time unit for train $j$ in cycle $k, \hat{x}_{j}(k+l \mid k)$ is the estimate of the departure time $x_{j}(k+l)$ in cycle $k+l$ based on the state of the network at the beginning of cycle $k$ and on the future inputs $u_{i, j}(k+p)$ for $p=0,1, \ldots, l$. The cost function $J_{\text {cost }}$ has three components: the first tries to keep the trains running on schedule, the second penalizes broken connections, whereas the third expresses the extra costs related to increasing the speeds of the trains. The factors $\lambda$ and $\mu$ determine the trade-off or the relative weights of the three components of the MPC cost function.

Now consider the following controller design problem - which is called the railway MPC problem at cycle $k$ :

$$
\min _{\substack{u_{i, j}(k), \ldots, u_{i, j}\left(k+N_{\mathrm{p}}-1\right) \\ v_{i}(k), \ldots, v_{i}\left(k+N_{\mathrm{p}}-1\right)}} J_{\text {cost }}(k)
$$

subject to

$$
\begin{aligned}
& \hat{x}_{j}(k+l \mid k)=\max \left(d_{j}(k+l),\right. \\
& \max _{i \in \mathscr{C}_{j}^{\text {hard }}(k+l)}\left(\hat{x}_{i}\left(k+l-\delta_{i, j}(k+l) \mid k\right)+a_{i}^{\text {nom }}(k+l)\right. \\
& \left.-v_{i}(k+l)+t_{i, j}^{\min }(k+l)\right), \\
& \max _{i \in \mathscr{C}_{j}^{\text {soft }}(k+l)}\left(\hat{x}_{i}\left(k+l-\delta_{i, j}(k+l) \mid k\right)+a_{i}^{\text {nom }}(k+l)\right. \\
& \left.\left.-v_{i}(k+l)+t_{i, j}^{\min }(k+l)-u_{i, j}(k+l)\right)\right) \\
& 0 \leqslant u_{i, j}(k+l) \\
& 0 \leqslant v_{i}(k+l) \leqslant a_{i}^{\text {nom }}(k+l)-a_{i}^{\min }(k+l) \\
& \text { for all } i, j \text { and for } l=0, \ldots, N_{\mathrm{p}}-1 \text { and with } \\
& \hat{x}_{j}(k+p \mid k)=x_{i}(k+p) \text { if } p<0 \text {. }
\end{aligned}
$$

In addition, to reduce the number of control variables we can - just as in conventional MPC - introduce a control horizon $N_{\mathrm{c}}\left(\leqslant N_{\mathrm{p}}\right)$ and set

$$
u_{i, j}(k+l)=u_{i, j}\left(k+N_{\mathrm{c}}-1\right) \text { and } v_{i}(k+l)=v_{i}\left(k+N_{\mathrm{c}}-1\right)
$$

for $l=N_{\mathrm{c}}, \ldots, N_{\mathrm{p}}-1$. This condition can be interpreted as follows: if after $N_{\mathrm{c}}$ cycles the delays have died out i.e., it is not necessary to break connections or to speed up the trains any more (so $u_{i, j}\left(k+N_{\mathrm{c}}\right)=v_{i}\left(k+N_{\mathrm{c}}\right)=0$ for all $i, j)$, - we do not break any connections or speed up trains in the subsequent cycles either. On the other hand, if the delays are still such that a connection should be broken in cycle $k+N_{\mathrm{c}}$ or such that some trains should still ride faster than usual, we will also break these connections or let the trains ride faster in the subsequent cycles.

Just like in conventional MPC we use a moving horizon approach, i.e., the railway MPC problem is solved for the current cycle, next the computed controls for that cycle are applied, and meanwhile the model is updated, and the computation is performed again for the next cycle. So we can also include predictable future delays (due to incidents, broken power lines, works, etc.) into our prediction model. 


\section{Algorithms for the railway MPC problem}

In general each step of the railway MPC problem leads to a nonconvex nonlinear optimization problem, which can be solved using a multi-start local optimization method such as multi-start sequential quadratic programming. A major disadvantage of local minimization is that in general the minimization routine will only return a local minimum and that several starting points are necessary to obtain a good approximation to the global optimum. Therefore, we now present an alternative approach to compute the optimal MPC control input, which is based on a mathematical programming problem called the Extended Linear Complementarity Problem (ELCP). The ELCP is defined as [2]:

Given $A \in \mathbb{R}^{p \times n}, c \in \mathbb{R}^{p}$, and $m$ subsets $\phi_{1}, \ldots, \phi_{m}$ of $\{1, \ldots, p\}$, find $z \in \mathbb{R}^{n}$ such that

$$
\prod_{i \in \phi_{j}}(A z-c)_{i}=0 \text { for } j=1, \ldots, m
$$

subject to $A z \geqslant c$.

Equation (13) can be interpreted as follows: each set $\phi_{j}$ corresponds to a group of inequalities of $A z \geqslant c$ and in each group at least one inequality should hold with equality. So for each $j$ there should exist an $i \in \phi_{j}$ such that $(A z-c)_{i}=0$.

Proposition 1 The evolution equations and the constraints of the railway MPC problem can be recast as an ELCP.

Proof: The proof follows the same lines as [6]. Although compared to [6] we have included extra control variables for the speed in this paper, these extra variables can be dealt with in the same way as the connection control variables as far as the conversion to the ELCP is concerned. Basically, the proof is based on the fact that $\max (\alpha, \beta)=\gamma$ with $\alpha, \beta, \gamma \in \mathbb{R}$ can be rewritten as the ELCP $\gamma-\alpha \geqslant 0$, $\gamma-\beta \geqslant 0,(\gamma-\alpha) \cdot(\gamma-\beta)=0$.

We discuss two approaches to compute the optimal railway MPC strategy using an ELCP.

\section{- optimization over the solution set of the ELCP:}

The solution set of an ELCP is the union of a subset of faces of the polyhedron defined by $A z \leqslant c$. In [2] we have developed an algorithm that yields a parametric description of the solution set of an ELCP in which each face is presented by its vertices. The optimal MPC strategy can now be obtained by determining for each face the point for which the objective function $J_{\text {cost }}(k)$ reaches a global minimum (this is an optimization over a convex set) and afterward selecting the overall minimum.

The advantage of this approach compared to straightforward nonlinear constrained optimization is that now we

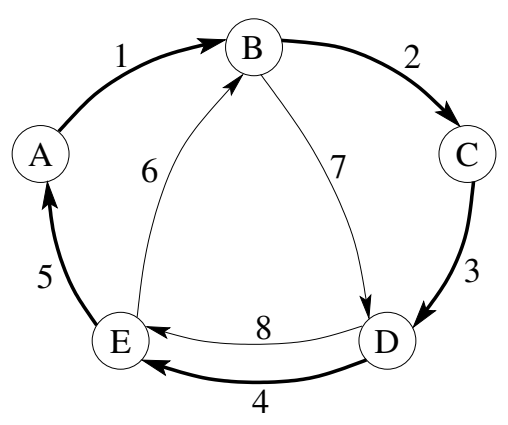

\section{Figure 3. The railroad network of the example of Section 5.}

have to solve a sequence of optimization problems with a convex feasible set instead of one big problem with a nonconvex feasible set. Optimization problems with a convex feasible set (albeit with a nonconvex objective function) are easier to solve numerically than problems with a nonconvex feasible set. Since the algorithm of [2] to compute the solution set of a general ELCP requires exponential execution times, this approach is not feasible if $N_{\mathrm{c}}$ is large. Therefore, we also present another ELCPbased approach, which uses mixed-integer optimization.

\section{- mixed-integer optimization:}

In [3] we show that an ELCP with a bounded feasible set can be rewritten as the following mixed-integer problem:

$$
\begin{array}{ll}
\delta \in\{0,1\}^{p}, z \in \mathbb{R}^{n} & \\
\sum_{i \in \phi_{j}} \delta_{i} \leqslant \# \phi_{j}-1 & \text { for } j=1,2, \ldots, m, \\
0 \leqslant(A z-c)_{i} \leqslant d_{i}^{\mathrm{upp}} \delta_{i} & \text { for } i=1, \ldots, p .
\end{array}
$$

with $d_{i}^{\text {upp }}=\max _{z \in \mathbb{R}^{n}}\left\{(A z-c)_{i} \mid A z \geqslant c\right\}$. This implies that the optimal MPC strategy can be determined by minimizing the objective function $J_{\text {cost }}(k)$ subject to (14)(16) using, e.g., a branch-and-bound method [8].

\section{Worked example}

Consider the railroad network of Figure 3. There are 5 stations (A, B, C, D and E), connected by 8 single tracks. There are two trains available. The first train follows the route $A \rightarrow B \rightarrow C \rightarrow D \rightarrow E \rightarrow A$ and the second train follows the route $B \rightarrow D \rightarrow E \rightarrow B$. The period of the timetable is $T=60$ minutes, Table 1 summarizes the information in connection with the nominal traveling times and the departure times. All the times are measured in minutes. The first cycle starts at time $t=0$. At the beginning of the first cycle the first train is in station A and the second in station B.

Suppose that we have to guarantee the following soft connection constraints: 


\begin{tabular}{|c|c|c|c|c|}
\hline Track & $\begin{array}{c}\text { From } \\
\text { station }\end{array}$ & $\begin{array}{c}\text { To } \\
\text { station }\end{array}$ & $\begin{array}{c}\text { Nominal } \\
\text { traveling } \\
\text { time }\end{array}$ & $\begin{array}{c}\text { Scheduled } \\
\text { departure time } \\
\text { modulo 60 }\end{array}$ \\
\hline 1 & A & B & 12 & 00 \\
2 & B & C & 10 & 15 \\
3 & C & D & 8 & 26 \\
4 & D & E & 6 & 36 \\
5 & E & A & 15 & 44 \\
6 & E & B & 26 & 45 \\
7 & B & D & 20 & 14 \\
8 & D & E & 6 & 37 \\
\hline
\end{tabular}

Table 1. The nominal traveling times and the departure times.

- the train on track 2 has to wait for the train on track 6 ,

- the train on track 6 has to wait for the train on track 4 ,

- the train on track 7 has to wait for the train on track 1.

The hard connection constraints are that the (virtual) trains on tracks 1, 2, 3, 4 and 5 are physically the same train, and the same holds for the trains on tracks 6,7 and 8 . The passengers get 2 minutes to change trains (for soft connections) and 1 minute to get out of the train (for hard connections).

First, we determine $x_{1}(k)$. At the beginning of the first cycle train 1 is in station A. So if $k=1$, the train departs from station A at time $t=0$. If $k>1$, the train departs from station $\mathrm{A}$ as soon as it has arrived in station A (via track 5) and the passengers have got the time to get out of the train and the earliest departure time indicated in the timetable has passed. Under nominal operations the train on track 1 in cycle $k$ will give connection to the train on track 5 that has departed in the previous cycle, i.e., in cycle $k-1$. This train arrives in station $\mathrm{A}$ at $t=x_{5}(k-1)+a_{5}(k)=x_{5}(k-1)+a_{5}^{\text {nom }}(k)-v_{5}(k)$, and afterward, the passengers have $t_{5,1}^{\min }(k)=1$ minute to get out of the train. The departure time of the train on track 1 according to the timetable is $0+(k-1) T$ in cycle $k$. So if we set $x_{5}(0)=-\infty$, we have

$$
x_{1}(k)=\max \left(x_{5}(k-1)+a_{5}^{\text {nom }}(k)-v_{5}(k)+1,0+(k-1) T\right)
$$

for $k=1,2, \ldots$ The train on track 1 will arrive in station B at $t=x_{1}(k)+a_{1}^{\text {nom }}(k)-v_{1}(k)$, after which the passengers have $t_{1,2}^{\min }(k)=1$ minute to get out of the train. If $k>1$, the train has to wait for the passengers of the train on track 6 , which arrives in station B at $t=x_{6}(k-1)+a_{6}^{\text {nom }}(k)-v_{6}(k)$. The passengers have $t_{6,2}^{\min }(k)=2$ minutes to change trains (since connection $6 \rightarrow 2$ is a soft connection). According to the timetable the train on track 2 can only depart after $t=$ $15+(k-1) T$. Furthermore, since the connection constraint is soft, we introduce a control variable $u_{6,2}(k)$ to break the connection if necessary. So if we set $x_{6}(0)=-\infty$, we have

$$
\begin{aligned}
x_{2}(k) & =\max \left(x_{1}(k)+a_{1}^{\text {nom }}(k)-v_{1}(k)+1,\right. \\
& \left.x_{6}(k-1)+a_{6}^{\text {nom }}(k)-v_{6}(k)+2-u_{6,2}(k), 15+(k-1) T\right)
\end{aligned}
$$

for $k=1,2, \ldots$ Similarly, we find

$$
\begin{gathered}
x_{3}(k)=\max \left(x_{2}(k)+a_{2}^{\text {nom }}(k)-v_{2}(k)+1,26+(k-1) T\right) \\
x_{4}(k)=\max \left(x_{3}(k)+a_{3}^{\text {nom }}(k)-v_{3}(k)+1,36+(k-1) T\right) \\
x_{5}(k)=\max \left(x_{4}(k)+a_{4}^{\text {nom }}(k)-v_{4}(k)+1,44+(k-1) T\right) \\
x_{6}(k)=\max \left(x_{4}(k)+a_{4}^{\text {nom }}(k)-v_{4}(k)+2-u_{4,6}(k),\right. \\
\left.x_{8}(k)+a_{8}^{\text {nom }}(k)-v_{8}(k)+1,45+(k-1) T\right) \\
x_{7}(k)=\max \left(x_{1}(k)+a_{1}^{\text {nom }}(k)-v_{1}(k)+2-u_{1,7}(k),\right. \\
\left.\quad x_{6}(k-1)+a_{6}^{\text {nom }}(k)-v_{6}(k)+1,14+(k-1) T\right) \\
x_{8}(k)=\max \left(x_{7}(k)+a_{7}^{\text {nom }}(k)-v_{7}(k)+1,37+(k-1) T\right)
\end{gathered}
$$

for $k=1,2, \ldots$ with $x_{j}(0)=-\infty$ for $j=1,2, \ldots, 8$.

Assume that all traveling times are equal to their usual values (cf. Table 1) except for those of the train on track 1 during the $1 \mathrm{st}, 2 \mathrm{nd}, 3 \mathrm{rd}$ and 4 th operation cycle: $a_{1}^{\text {nom }}(1)=$ $a_{1}^{\text {nom }}(2)=25, a_{1}^{\text {nom }}(3)=20$, and $a_{1}^{\text {nom }}(4)=15$.

Now we apply MPC algorithm (including the receding horizon approach) to the system over a simulation period $[1, K]$ with $K=12$. We take $N_{\mathrm{c}}=0.5 n=4, N_{\mathrm{p}}=6, \lambda=\mu=$ $0.5, c_{6,2}^{\text {broken }}(k)=2, c_{1,7}^{\text {broken }}(k)=c_{4,6}^{\text {broken }}(k)=5, c_{i, j}^{\text {off }}(k)=1$, $c_{i}^{\text {speed }}(k)=1$, and $a_{i}^{\text {nom }}(k)-a_{i}^{\min }(k)=1$ (i.e., the maximal possible gain in the traveling time by running at full speed is 1 minute) for all $i, j, k$. We discern four different cases: no control (NC), connection control only (CC), speed control only (SC), and connection and speed control (C\&SC).

We have assumed that at the beginning of each MPC cycle both the correct state vector (i.e., the actual departure times in the previous cycle) and the delay for that cycle were known ${ }^{1}$. By re-computing the optimal control strategy at the beginning of each cycle and by applying the receding horizon approach, MPC can adapt the control strategy to changes in the system and take new information (e.g., about delays and actual departure and arrival times) into account as it becomes available. Clearly, this has a positive effect on the performance of the system as can be seen from Figure 4 and Table 2, where we have also listed the "total" cost function over the simulation horizon:

$$
\begin{aligned}
J_{\text {total }}= & \sum_{l=1}^{K} \sum_{j=1}^{n}\left(x_{j}(l)-d_{j}(l)\right)^{2}+\lambda \sum_{l=1}^{K} \sum_{j=1}^{n} \sum_{i \in \mathscr{C}_{j}^{\text {soft }}(l)} J_{i, j}^{\text {broken }}(l)+ \\
& \mu \sum_{l=1}^{K} \sum_{j=1}^{n} \sum_{i \in \mathscr{C}_{j}(l)} J_{i}^{\text {speed }}(l) .
\end{aligned}
$$

Finally, we have computed the optimal control (OC) signal that minimizes $J_{\text {total }}$ by solving the MPC optimization problem for cycle $k=1$ with all delays assumed to be known and with $N_{\mathrm{c}}=N_{\mathrm{p}}=K$. In this particular case, the performance of the OC control signal coincides almost completely with that of the closed-loop MPC signal. In general, the OC control signal may lead to a better performance (under

\footnotetext{
${ }^{1}$ I.e., for $k=1$ we know that $a_{1}^{\text {nom }}(1)=25$ but the other values are assumed to be given by Table 1 , for the optimization for $k=2$ we assume that $a_{1}^{\text {nom }}(2)=25$, with the other values given by Table 1 , etc.
} 


\begin{tabular}{|l|l|r|}
\hline Case & \multicolumn{1}{|c|}{ Delays } & \multicolumn{1}{|c|}{$J_{\text {total }}$} \\
\hline No control (NC) & $13,22,26,25,21,18,14,11,7,4,0,0$ & 19333.00 \\
Connection (CC) & $11,20,24,23,21,17,13,9,5,1,0,0$ & 10768.90 \\
Speed (SC) & $12,16,15,9, \quad 1,0,0,0,0,0,0,0$ & 2973.33 \\
Connection and speed (C\&SC) & $10,14,13,7, \quad 1,0,0,0,0,0,0,0$ & 1567.97 \\
Optimal control (OC) & $10,14,13,7,1,0,0,0,0,0,0,0$ & 1568.01 \\
\hline
\end{tabular}

Table 2. Delays and total cost function for the closed-loop MPC and OC control signals.

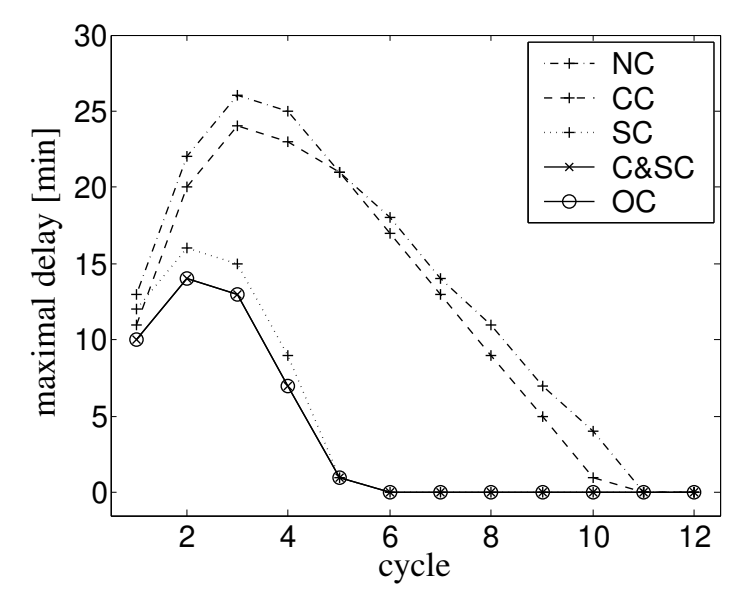

Figure 4. Delays for the closed-loop MPC and OC control signals over $[1, K]$.

the assumption that there are no disturbances and modeling errors). Note, however, that computing the OC control input requires much more computation time than computing the MPC control inputs. Furthermore, the MPC approach is more robust since it is a control strategy that can take changes in the system and new information about actual arrival and departure times, actual traveling times, and updated estimates of the delays into account.

\section{Conclusions}

We have proposed a modeling framework for railway and subway systems with both soft and hard synchronization constraints, and we have presented an MPC-like control design method for these systems. The control actions consist in breaking certain soft connections or letting trains ride faster to prevent delays from accumulating, but this can only be done at a certain cost. We have shown that the resulting optimization problem can be solved using ELCPs. Furthermore, due to the use of a moving horizon strategy and a control horizon the railway MPC method can be used in online applications and it can deal with (predicted) changes in the system parameters. So if we can predict the delays that will occur due to an incident, maintenance, or works, we can include this information when determining the optimal control input for the next operation cycles of the network.

\section{Acknowledgment}

Research partially funded by the Dutch Technology Foundation STW project "Model predictive control for hybrid systems" (DMR.5675).

\section{References}

[1] J. Braker. Max-algebra modelling and analysis of time-table dependent transportation networks. In Proc. 1st European Control Conf., pages 1831-1836, Grenoble, July 1991.

[2] B. De Schutter and B. De Moor. The extended linear complementarity problem. Mathematical Programming, 71(3):289325, Dec. 1995.

[3] B. De Schutter, W. Heemels, and A. Bemporad. On the equivalence of linear complementarity problems. To appear in Operations Research Letters.

[4] B. De Schutter and T. van den Boom. Model predictive control for discrete-event systems with soft and hard synchronization constraints. In Proc. Workshop on Max-Plus Algebras and Their Applications to Discrete-Event Systems, Theoretical Computer Science, and Optim., Prague, Aug. 2001.

[5] B. De Schutter and T. van den Boom. Model predictive control for max-plus-linear discrete event systems. Automatica, 37(7):1049-1056, July 2001.

[6] B. De Schutter and T. van den Boom. Model predictive control for railway networks. In Proc. 2001 Int. Conf. on Adv. Intelligent Mechatronics, pages 105-110, Como, July 2001.

[7] P. de Waal, A. Overkamp, and J. van Schuppen. Control of railway traffic on a single line. In Proc. European Control Conf. (ECC'97), Brussels, paper 230, July 1997.

[8] R. Fletcher and S. Leyffer. Numerical experience with lower bounds for MIQP branch-and-bound. SIAM Journal on Optimization, 8(2):604-616, May 1998.

[9] B. Heidergott and R. de Vries. Towards a (max,+) control theory for public transportation networks. Discrete Event Dynamic Syst.: Theory \& Appl., 11(4):371-398, Oct. 2001.

[10] J. Maciejowski. Predictive Control with Constraints. Prentice Hall, 2002.

[11] R. Minciardi, M. Paolucci, and R. Pesenti. Generating optimal schedules for an underground railway line. In Proc. 34th IEEE Conf. on Decision and Control, pages 4082-4085, New Orleans, Dec. 1995.

[12] A. Soeterboek. Predictive Control - A Unified Approach. Prentice Hall, 1992. 